\title{
Avaliação do controle da asma em pacientes acompanhados em dois ambulatórios de pneumologia vinculados à Estratégia Saúde da Família em Tubarão, Santa Catarina, Brasil
}

\author{
Assessment of asthma control in patients monitored at two pulmonology outpatient clinics linked to the Family Health \\ Strategy in Tubarão, Santa Catarina state, Brazil

\section{Evaluación del control del asma en pacientes asistidos en dos ambulatorios de neumología vinculados a la Estrategia de Salud Familiar en Tubarão, Santa Catarina, Brasil}

Daniel de Andrade Hygidio. Universidade do Sul de Santa Catarina (UNISUL). Tubarão, SC, Brasil. daniel.hygidio@gmail.com (Autor correspondente) Jane da Silva. Universidade do Sul de Santa Catarina (UNISUL). Florianópolis, SC, Brasil. janedasilva1808@gmail.com

Sérgio Silveira. Universidade do Sul de Santa Catarina (UNISUL). Tubarão, SC, Brasil. serginhoo_@hotmail.com

Beatriz Camisão Schwinden. Universidade do Sul de Santa Catarina (UNISUL). Tubarão, SC, Brasil. biics_@gmail.com

\section{Resumo}

Introdução: A asma é uma doença crônica debilitante, que pode gerar restrições diretamente ligadas a um mau controle da doença. 0 controle da asma pode ser avaliado por instrumentos padronizados. Objetivo: Determinar o controle da asma por meio do Questionário de Controle da Asma (QCA) e do Teste de Controle da Asma (TCA) e a classificação de acordo com as Diretrizes para o Manejo da Asma, de 2012. Métodos: Estudo observacional transversal com pacientes atendidos em ambulatórios de referência vinculados à Estratégia Saúde da Família em Tubarão-SC. A população foi composta por todos os pacientes asmáticos adultos que recorreram a consultas médicas durante o período de janeiro a novembro de 2010. 0s dados foram compilados em uma planilha Excel e analisados pelo programa INSTAT 3. Foi realizada análise descritiva e aplicado teste quiquadrado para verificar associação entre respostas do TCA e QCA. Resultados: Foram avaliados 49 pacientes, tendo, a maioria deles, asma intermitente ou persistente leve (51\%). Na avaliação do controle da asma o TCA mostrou que 69,3\% dos pacientes não estavam controlados. Em relação ao QCA, foram considerados fora do alvo 75,5\% dos pacientes. Houve associação significativa entre o TCA e QCA ( $p<0,05)$. Quanto às Diretrizes para o Manejo da Asma, 2012, $57,14 \%$ dos pacientes foram considerados não controlados; 30,6\% estavam parcialmente controlados; e 12,2\%, controlados. Conclusão: Os asmáticos estavam, em sua maioria, não controlados. Torna-se necessário identificar os fatores ligados ao descontrole e buscar estratégias, visando melhorar os escores para redução da morbimortalidade associada à asma.

\footnotetext{
Abstract

Introduction: Asthma is a chronic debilitating disease which can generate constraints directly associated with poor disease control. Asthma control can be evaluated using standardized instruments. Objective: To determine asthma control through the Asthma Control Questionnaire (ACQ) and Asthma Control Test (ACT), and to classify it according to the Guidelines for the Management of Asthma, 2012. Methods: This is a cross-sectional observational study with patients of two pulmonology outpatient clinics linked to the Family Health Strategy in the municipality of Tubarão, Santa Catarina state. The population consisted of all adult asthmatic patients who resorted to medical appointments from January to November 2010. The data were compiled in Excel and analyzed by INSTAT 3 software. We performed descriptive analysis and applied the chi-square test to assess the association between ACT and ACQ answers. Results: We evaluated 49 patients, most of them presenting intermittent or mild persistent asthma (51\%). In the assessment of asthma control, ACT showed that $69.3 \%$ of patients were considered uncontrolled, while ACQ showed that $75.5 \%$ of patients were considered off-target. Significant association was found between ACT and ACQ $(p<0.05)$. Regarding the 2012 Guidelines for the Management of Asthma, $57.14 \%$ of patients were considered uncontrolled; $30.6 \%$ were partially controlled; and $12.2 \%$ were controlled. Conclusion: Most asthmatics were uncontrolled. It is necessary to identify the factors related to uncontrolled patients and search strategies to improve health scores for reduction of morbimortality caused by asthma.
}

Palavras-chave:

Asma Prevenção e Controle Questionários

Keywords: Asthma Prevention and Control Questionnaires
Fonte de financiamento: Programa Universitário de Iniciação Científica da Unisul. Parecer CEP: Unisul 10.621.4.01.III. 


\section{Resumen}

Introducción: El asma es una enfermedad crónica debilitante, que puede generar restricciones directamente relacionadas con el mal control de la enfermedad. El control del asma puede ser evaluado utilizando instrumentos estandarizados. Objetivo: Determinar el control del asma a través del Cuestionario de Control del Asma (CCA) y la Prueba de Control del Asma (PCA) y la clasificación de acuerdo con la Guía para el Manejo del Asma, de 2012. Métodos: Estudio observacional transversal con pacientes atendidos en ambulatorios de referencia vinculados a la Estrategia de Salud Familiar en Tubarão-SC. La población estaba compuesta por todos los pacientes asmáticos adultos que recurrieron a citas médicas durante el período de enero a noviembre de 2010. Los datos se recopilaron en una hoja de cálculo Excel y se analizaron con el software INSTAT 3. Se realizó un análisis descriptivo y se aplicó el test chi-cuadrado para evaluar la asociación entre respuestas del PCA y CCA. Resultados: Se evaluaron 49 pacientes, la mayoría de ellos con asma intermitente o persistente leve (51\%). En la evaluación del control del asma, el PCA mostró que el 69,3\% de los pacientes no estaban bajo control. En el CCA se consideró fuera de objetivo al 75,5\% de los pacientes. Se encontró una asociación significativa entre PCA y CCA ( $p<0,05)$. En cuanto a la Guía para el Manejo del Asma, de 2012, se consideró al 57,14\% de los pacientes fuera de control; el 30,6\% estaban parcialmente bajo control; y el 12,2\% bajo control total. Conclusión: La mayoría de los asmáticos no estaban bajo control. Es necesario identificar los factores relacionados con el descontrol y buscar estrategias con el objetivo de mejorar los puntajes para reducir la morbimortalidad asociada al asma.
Palabras clave:

Asma

Prevención y Control

Cuestionarios

\section{Introdução}

A visão mais recente sobre a asma a caracteriza como um distúrbio complexo composto por diferentes subtipos (fenótipos) e com causas diversas. O conceito clássico que relaciona asma à inflamaçáo, hiperresponsividade brônquica, broncoconstrição e sintomas ainda permanece nos consensos e nas diretrizes. No entanto, tem-se buscado ampliar essa definição ao inserir a ideia de que asma é uma síndrome clínica heterogênea que responde aos tratamentos de diferentes maneiras. ${ }^{1}$

É uma doença de alta prevalência e morbidade nos dias atuais, sendo responsável por atingir, em média, 300 milhões de pessoas no mundo, segundo a Organização Mundial da Saúde (OMS). ${ }^{2}$ No Brasil, assim como em outros países em desenvolvimento, sua incidência vem aumentando, sendo responsável por 160 mil internaçôes, constituindo-se na quarta maior causa de internaçóes pelo Sistema Único de Saúde (SUS). ${ }^{3}$

A asma acomete crianças e adultos e, assim como outras doenças crônicas debilitantes, interfere de forma complexa no bem-estar dos pacientes, sendo responsável por um alto índice de abstenção escolar e no trabalho, causando, em diferentes graus, limitaçôes físicas, emocionais e sociais. Estas restrições estão diretamente ligadas a um mau controle da doença e à forma de exacerbação súbita diante dos estímulos, gerando constante tensão e desconfiança no sujeito acometido. ${ }^{4}$

A definição do controle da doença é obtida, segundo a Global Initiative for Asthma (GINA) e pela Sociedade Brasileira de Pneumologia e Tisiologia, ${ }^{5}$ seguindo determinados parâmetros clínicos e de função respiratória. Tais parâmetros podem ser mensurados por dois instrumentos quantitativos, elaborados em consensos e validados no Brasil, ambos disponíveis para a avaliação do nível de controle da asma. Denominam-se Teste de Controle da Asma (TCA ou ACT) e Questionário de Controle da Asma (QCA ou ACQ). Estes instrumentos, além de possuírem uma boa sensibilidade discriminativa entre pacientes com e sem asma bem controlada, são autoaplicáveis, possuem uma fácil linguagem, possibilitando o entendimento das questốes propostas ${ }^{6,7}$ e podem ser aplicados na atenção primária à saúde (APS) em crianças $^{8}$ e adultos. ${ }^{9}$

De modo geral, os índices de controle da asma sáo conhecidamente baixos, sendo necessário que estes sejam modificados. Assim, conhecer a dimensão do controle da asma é fundamental para serem criadas estratégias de manejo da doença, visando o controle da maioria dos pacientes.

Os ambulatórios públicos de Tubarão-SC encaminham para avaliação periódica especializada os pacientes com asma, e não havia no município dados referentes ao controle de pacientes asmáticos, dificultando a estimativa pelos profissionais de saúde de suas açóes diante da doença. Desse modo, o objetivo do presente estudo é avaliar o perfil de pacientes asmáticos encaminhados a partir da Estratégia Saúde da Família (ESF) aos ambulatórios médicos de especialidade (AMEs), vinculados ao SUS e aplicar-lhes os questionários QCA e TCA, para avaliação do controle da doença.

\section{Métodos}

Foi realizado um estudo observacional transversal nos dois ambulatórios médicos de especialidade (AME) em Pneumologia vinculados à ESF de Tubarão, SC.

A população foi composta por um censo de pacientes asmáticos adultos que recorreram a consultas médicas durante o período de janeiro a dezembro de 2010 e que concordaram em participar do estudo. 
Para a identificação dos casos, foi feita uma análise retrospectiva das consultas realizadas no ano de 2010, por meio do Boletim de Informações Diárias de Atendimento Médico (BIDAM). Com a autorização do responsável pelo serviço ambulatorial, obteve-se o meio de contato do paciente asmático. Foram prestadas orientaçóes sobre o estudo, fez-se o convite à participação e foram dadas explicaçóes sobre o Termo de Consentimento Livre e Esclarecido (TCLE). Para aqueles que aceitavam participar, era agendado um horário de atendimento no AME de Alergia e Pneumologia. Os pacientes assinavam o TCLE, era aplicado um questionário de perfil do paciente e, após orientaçôes sobre os instrumentos de avaliação de controle da asma, o TCA e QCA eram preenchidos pelos pacientes. Durante o preenchimento, caso houvesse dúvidas, o pesquisador prestava esclarecimentos adicionais, sem induzir o paciente a nenhum padrão específico de resposta. Em seguida, era realizada espirometria com prova de broncodilatação.

Foram excluídos pacientes impossibilitados de preencher os questionários por incapacidade de compreensão, fumantes e/ou com doença pulmonar obstrutiva crônica (DPOC), ou aqueles sem condiçóes de realização de espirometria.

Os dados coletados foram compilados em uma planilha Excel e analisados pelo programa INSTAT 3. Os resultados das características clínicas e socioeconômicas dos pacientes foram apresentados em valores absolutos e percentuais. Foi aplicado o teste quiquadrado para verificar associaçáo de respostas do TCA e QCA, sendo considerado significativo o valor de $\mathrm{p}<0,05$ e também foi utilizado o teste de Pearson para avaliar a correlação entre os questionários. Os resultados obtidos com o QCA e o TCA foram equiparados aos dados da tabela de classificação de controle da asma das Diretrizes de Manejo da Asma (2012). ${ }^{5}$

O projeto foi aprovado pelo comitê de ética em pesquisa da Universidade do Sul de Catarina (Unisul), parecer consubstanciado número 10.621.4.01.III.

\section{Resultados}

Foram encontrados registros de 68 pacientes com asma. No entanto, 4 não aceitaram participar da pesquisa; não se conseguiu contato com 5; 1 havia falecido; e 10 foram excluídos devido a tabagismo e/ou DPOC associado (8 pacientes) ou por terem usado medicaçáo broncodilatadora antes de realizar espirometria ( 2 pacientes). Assim, foram investigados 49 asmáticos ( $72 \%$ dos registros, $81 \%$ dos que aceitaram participar). Os dados sociodemográficos desses pacientes estáo apresentados na Tabela 1.

Tabela 1. Características demográficas e socioeconômicas de asmáticos acompanhados em dois ambulatórios de Tubarão-SC, 2010.

\begin{tabular}{|c|c|c|}
\hline Características & $\mathrm{N}$ & $\mathrm{n} \%$ \\
\hline Média de Idade (Min-máx.) & $51,4(18-76)$ & \\
\hline $\begin{array}{l}<20 \text { anos } \\
21-30 \text { anos } \\
31-40 \text { anos } \\
41-50 \text { anos } \\
51-60 \text { anos } \\
61-70 \text { anos } \\
71-80 \text { anos }\end{array}$ & $\begin{array}{c}2 \\
4 \\
3 \\
12 \\
14 \\
9 \\
5\end{array}$ & $\begin{array}{c}4 \\
8 \\
6,1 \\
24,5 \\
29 \\
18,4 \\
10\end{array}$ \\
\hline $\begin{array}{l}\text { Mexo } \\
\text { Masculino } \\
\text { Feminino }\end{array}$ & $\begin{array}{l}17 \\
32\end{array}$ & $\begin{array}{l}34,7 \\
65,3\end{array}$ \\
\hline $\begin{array}{l}\text { Etnia } \\
\text { Caucasiano } \\
\text { Negro }\end{array}$ & $\begin{array}{c}34 \\
3\end{array}$ & $\begin{array}{c}91,8 \\
8,2\end{array}$ \\
\hline $\begin{array}{l}\quad \text { Escolaridade } \\
\text { Básico } \\
\text { Fundamental } \\
\text { Médio } \\
\text { Superior } \\
\text { Não escolarizado }\end{array}$ & $\begin{array}{c}11 \\
16 \\
12 \\
2 \\
4\end{array}$ & $\begin{array}{r}24,4 \\
35,5 \\
26,6 \\
4,4 \\
8,8\end{array}$ \\
\hline $\begin{array}{l}\text { Ecupação } \\
\text { Empregado } \\
\text { Desempregado } \\
\text { Aposentado }\end{array}$ & $\begin{array}{c}13 \\
6 \\
24\end{array}$ & $\begin{array}{l}30,2 \\
13,9 \\
55,8\end{array}$ \\
\hline $\begin{array}{l}>2 \text { s.m. }{ }^{*} \quad \text { Renda } \\
<2 \text { s.m. }\end{array}$ & $\begin{array}{l}11 \\
30\end{array}$ & $\begin{array}{l}26,8 \\
73,1\end{array}$ \\
\hline
\end{tabular}

*s.m. (salários mínimos). 
Na Tabela 2, observam-se as características clínicas dos pacientes asmáticos avaliados.

Os resultados obtidos no Teste de Controle da Asma (TCA) e no Questionário de Controle da Asma (QCA) dos pacientes asmáticos estudados são apresentados na Tabela 3.

Buscou-se relacionar as respostas de ambos os instrumentos TCA e QCA. A Figura 1 apresenta tal relação.

Tabela 2. Características clínicas de asmáticos acompanhados em dois ambulatórios de Tubarão-SC, 2010.

\begin{tabular}{|c|c|c|}
\hline Características & $\mathrm{N}$ & $\mathrm{n} \%$ \\
\hline \multicolumn{3}{|l|}{ Medicamentos utilizados } \\
\hline $\mathrm{Cl}^{\mathrm{a}}$ & 3 & 6,2 \\
\hline $\mathrm{Cl}$ associado à $\mathrm{LABA}^{\mathrm{b}}$ & 28 & 58,3 \\
\hline BD Beta-2 de curta ação & 11 & 22,9 \\
\hline Nenhum & 6 & 12,5 \\
\hline \multicolumn{3}{|l|}{ Gravidade da Asma } \\
\hline Intermitente & 8 & 16,3 \\
\hline Persistente Leve & 17 & 34,7 \\
\hline Persistente Moderada & 13 & 26,5 \\
\hline Persistente Grave & 11 & 22,4 \\
\hline $\begin{array}{l}\text { Duração dos sintomas de asma em média de anos } \\
\text { (Mínimo/Máximo/Média/DPd) }\end{array}$ & 25,92 (2/61/26/17,6) & \\
\hline
\end{tabular}

Tabela 3. Controle da asma de acordo com os resultados obtidos nos testes aplicados aos asmáticos em dois ambulatórios de Tubarão-SC, 2010.

\begin{tabular}{lcc}
\multicolumn{1}{c}{ Controle } & $\mathrm{N}$ & $\mathrm{n} \%$ \\
\hline Controle da Asma segundo TCA: & & \\
Controlados & 15 & 30,6 \\
Não Controlados & 34 & 69,3 \\
Controle da Asma segundo QCA: & & \\
Controlados & 12 & 24,5 \\
Não Controlados & 37 & 75,5 \\
Classificação das diretrizes de 2012: & & \\
Controlados & 6 & 12,2 \\
Parcialmente controlados & 15 & 30,6 \\
Não controlados & 28 & 57,1 \\
\hline
\end{tabular}

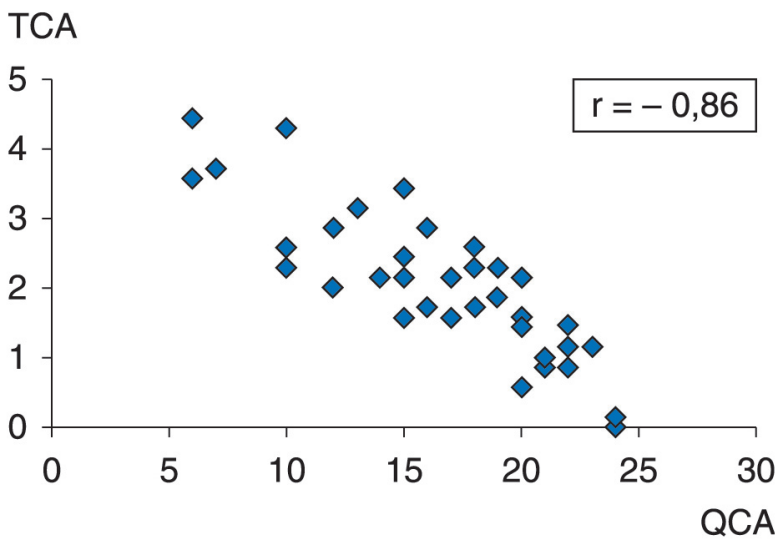

Figura 1. Relação entre respostas observadas nos instrumentos de avaliação de controle da asma aplicados aos pacientes acompanhados em dois ambulatórios de Tubarão-SC, 2010. 


\section{Discussão}

Observa-se que os pacientes asmáticos que procuraram acompanhamento no SUS por meio dos dois serviços ambulatoriais da Universidade do Sul de Santa Catarina apresentavam idade média de 51,4 anos e eram em sua maioria mulheres (65,3\%). Este indicador realça a importância da existência de um serviço organizado que tenha como foco o acompanhamento sistemático dos pacientes, uma vez que a maioria dos pacientes se encontra com uma média de idade elevada, podendo a asma estar acompanhada de comorbidades acumuladas ao longo da vida. Percebe-se que o sexo feminino, majoritário na pesquisa, reafirma estudos já produzidos que revelam a busca mais frequente da mulher por cuidados com a saúde. ${ }^{10}$

Quanto à cor da pele predominante, esta se constituiu de pessoas caucasianas $(91,8 \%)$, contrapondo-se com uma pequena proporção de negros $(8,2 \%)$, com nível de escolaridade em sua maioria de ensino fundamental $(35,5 \%)$ e de ensino médio $(26,6 \%)$. Chama a atenção do pesquisador o percentual de pacientes caucasianos, o que corrobora com dados do Censo 2010 que demonstram menores índices de população negra do Brasil na região Sul.

Outro dado relevante está relacionado à condição econômica dos pacientes, em sua maioria aposentados $(55,8 \%)$ ou empregados $(30,2 \%)$ e com renda inferior a dois salários mínimos $(73,1 \%)$. Os dados vão ao encontro das características de pessoas que tem o SUS como única possibilidade de acessar o direito de cuidados com a saúde. Articulando este dado com o nível de escolaridade (que somam ensino fundamental e médio) pode-se considerar que a população que recorreu aos serviços é aquela que tem acesso a mínimas informaçôes e oportunidades de tratamento. Esta consideração é preliminar e exige um estudo mais apurado da realidade.

Estudos sobre a prevalência de asma são largamente realizados na população de crianças e adolescentes e mostram uma variação nos diferentes locais avaliados. ${ }^{11-13}$ Dados recentes obtidos por um estudo multicêntrico (International Study for Asthma and Allergies in Childhood - ISAAC) demonstraram que, no Brasil, este índice é de 20\% para a faixa etária de treze a quatorze anos. ${ }^{14}$ Segundo a IV Diretriz Brasileira para o Manejo da Asma, aproximadamente em $60 \%$ dos casos a doença manifesta-se de forma intermitente ou persistente leve e em $25 \%$ como moderada. ${ }^{15}$ Resultados semelhantes foram vistos no presente estudo, em que $51 \%$ dos pacientes estudados tinham asma intermitente ou persistente leve e $26,5 \%$ apresentavam asma persistente moderada.

As avaliaçôes da GINA preconizam que a meta primária no manejo da asma seria alcançar um bom controle da doença. ${ }^{2}$ É no contexto de melhor avaliar esses parâmetros, visando a não subestimação ou superestimação do quadro relatado pelo paciente, que o TCA e o QCA inserem-se.

O TCA desenvolvido por Nathan et al. ${ }^{6}$ consiste em um teste simples e facilmente aplicável nas unidades de atenção primária, visto que não necessita da análise da função pulmonar. Em sua validação para o Brasil, teve como base de corte 18 pontos, mostrando elevados valores de sensibilidade $(92,8 \%)$, especificidade $(85,7 \%)$, valor preditivo negativo $(86,0 \%)$ e positivo $(85,7 \%)$.

O QCA, idealizado por Juniper et al. ${ }^{7}$ em 1999, possui em sua análise um ponto de corte usado em ensaios clínicos de 1,5 possuindo também bons parâmetros de sensibilidade (91\%), especificidade (64\%), valor preditivo negativo (80\%) e positivo (81\%).

Entre os asmáticos avaliados neste estudo, foram encontrados $69,3 \%$ de pacientes não controlados segundo o teste. Embora à primeira vista estes números possam surpreender, quando comparados a um programa de controle de asma de referência nacional, $53 \%$ de um total de 289 asmáticos apresentaram doença não controlada ${ }^{16}$ reafirmando expectativas internacionais que mostram que $54,1 \%$ dos asmáticos apresentam-se não controlados. ${ }^{17,18}$

Em relação ao questionário, os pacientes considerados fora do alvo perfizeram 75,5\%. Utilizando-se como base os parâmetros de controle clínico atual, fornecidos nas últimas Diretrizes da Sociedade Brasileira de Pneumologia e Tisiologia para o Manejo da Asma-2012, 57,14\% dos pacientes foram considerados não controlados; 30,6\% como parcialmente controlados e 12,2\% considerados controlados. Esta menor porcentagem de pacientes náo controlados obtidos por este parâmetro se deve a sua forma de avaliação que aloca grande parte destes pacientes como parcialmente controlados.

Dados de literatura alertam que a asma é uma das condiçóes mais sensíveis às açôes da atenção primária à saúde que visam diminuir o número de internaçóes evitáveis. ${ }^{19}$ Diante dos dados apresentados neste artigo, fica nítida a real necessidade da criaçáo de um programa efetivo focado na asma na ESF, com o intuito de melhorar esses indicadores e, com isso, evitar as complicações advindas do mau controle da doença. 
Houve correlação significativa $(\mathrm{p}<0,01)$ nas respostas encontradas nos instrumentos de avaliação do controle TCA e QCA, como mostra a Figura 1.

Houve também pouca variação na discriminaçáo entre controlados e não controlados, de acordo com as respostas aos instrumentos. Pequenas discrepâncias podem ser decorrentes dos menores índices de especificidade do questionário ou pelos seus padrōes de resposta, por vezes, ainda pouco claros à população com baixo índice de escolaridade, segundo padrōes internacionais, no entanto isso não interferiu na análise da avaliação do controle da asma, de modo geral.

\section{Conclusões}

O controle da asma em pacientes atendidos em dois ambulatórios públicos de Tubarão-SC está aquém do preconizado pelas novas diretrizes internacionais, entretanto, assemelha-se a resultados de estudos tanto nacionais quanto internacionais.

Os parâmetros usados na prática clínica, em uma avaliação superficial, podem classificar equivocadamente um paciente pouco controlado como sendo bem controlado, portanto, os métodos quantitativos do TCA ou QCA e da tabela de controle do consenso de asma demonstraram ser mais discriminativos e correspondentes, de modo geral.

$\mathrm{Na}$ atenção primária à saúde, em especial, destaca-se a função do teste de controle da asma devido à sua rápida execução e total dispensa da espirometria para a avaliaçáo do controle da doença, o que permite sua maior reprodutibilidade.

Assim, independentemente do instrumento aplicado, a avaliação do controle da asma deveria ser o primeiro passo a ser realizado, em qualquer nível de assistência ao paciente asmático. Torna-se necessário buscar os fatores ligados ao descontrole para, diante da realidade apresentada, elaborar-se estratégias visando à melhoria dos escores que repercutirão na redução da morbimortalidade da doença.

\section{Referências}

1. Silva J, Oliveira F. Asma como diagnosticar e tratar. RBM. ed. especial 2012 Dec; 69(12): 83-96. [Internet]. Disponível em: http://www.moreirajr.com. $\mathrm{br} /$ revistas.asp?fase=r003\&id_materia $=529$.

2. Global Initiative for Asthma. Guidelines: GINA report, Global Strategy for Asthma Management and Prevention, 2012 [online] $110 \mathrm{p}$. [acesso em 2013 mar]. Disponível em: http://www.ginasthma.org/local/themes/gina/images/document-download-text.png

3. Brasil. Ministério da Saúde do Brasil. Departamento de Informática do SUS [online]. Brasília: DATASUS [acesso em 2013 mar]. Morbidade hospitalar do SUS - por local de internação - Brasil. Disponível em: http://tabnet.datasus.gov.br/cgi/tabcgi.exe?sih/cnv/miuf.def

4. Juniper EF. Assessing asthma quality of life: its role in clinical practice. Breathe. 2005; 1(3): 192-205.

5. Sociedade Brasileira de Pneumologia e Tisiologia. Diretrizes da Sociedade Brasileira de Pneumologia e Tisiologia para o Manejo da Asma. J Bras Pneumol. 2012; 38(Supl. 1): S1-46.

6. Roxo JPF, Ponte EV, Ramos DCB, Pimentel L, D’Oliveira A Jr, Cruz AA. Validação do Teste de Controle da Asma em português para uso no Brasil. J Bras Pneumol. 2010; 36(2): 159-66. http://dx.doi.org/10.1590/S1806-37132010000200002

7. Leite M, Ponte EV, Petroni J, D’Oliveira A Jr, Pizzichini E, Cruz A. Avaliação do Questionário de Controle da Asma validado para uso no Brasil. J Bras Pneumol. 2008; 34(10): 756-63. http://dx.doi.org/10.1590/S1806-37132008001000002

8. Liu A, Gilesenan A, Stanford R, Lincourt W, Ziemiecki W, Ortega H. Status of Asthma Control in Pediatric Primary Care: Results From the Pediatric Asthma Control Characteristics and Prevalence Survey Study. J. Pediatr. 2010; 157(2): 276-81. http://dx.doi.org/10.1016/j.jpeds.2010.02.017

9. Zhou X, Ding FM, Lin JT, Yin KS. Validity of Asthma Control Test for Asthma Control Assessment in Chinese Primary Care Settings. Chest. 2009; 135(4): 904-10. http://dx.doi.org/10.1378/chest.08-0967

10. Ribeiro MCSA, Barata RB, Almeida MF, Silva ZP. Perfil sociodemográfico e padrão de utilização de serviços de saúde para usuários e não-usuários do SUS - PNAD 2003. Ciênc Saúde Coletiva. 2006; 11(4) 1011-22.

11. Weitzman M, Gortmaker SL, Sobol AM, Perrin JM. Recent trends in the prevalenceand severity of childhood asthma. JAMA. 1992; (268): 2673-7. http://dx.doi.org/10.1001/jama.1992.03490190073034

12. Garcia-Marcos L, Quiros AB, Hernandez GG. Stabilization of asthma prevalence among adolescents and increase among schoolchildren (ISAAC phases I and III) in Spain. Allergy. 2004; (59): 1301-7. http://dx.doi.org/10.1111/j.1398-9995.2004.00562.x

13. Braun-Fahrlander C, Gassner M, Grize L. No further increase in asthma, hay fever and atopic sensitisation in adolescents living in Switzerland. Eur Respir J. 2004; (23): 407-13. http://dx.doi.org/10.1183/09031936.04.00074004 
14. Beasley R, Keil U, Mutius E, Pearce N. Worldwide variation in prevalence of symptoms of asthma, allergic rhinoconjuntivitis, and atopic eczema: ISAAC. The International Study of Asthma and Allergies in Childhood (ISAAC) Steering Committee. Lancet. 1998; (351): 1225-32. http://dx.doi. org/10.1016/S0140-6736(97)07302-9

15. Stirbulov R. IV Diretrizes Brasileiras para o Manejo da Asma. J Bras Pneumol. 2006; 32 (7): 447-74.

16. Pizzichini E. Defining asthma control: time to look for new definitions? J Bras Pneumol. [online]. 2007; 33(6) Dez. http://dx.doi.org/10.1590/S180637132007000600002

17. Rabe KF, Adachi M, Lai CK, Soriano JB, Vermeire PA, Weiss KB, et al. Worldwide severity and control of asthma in children and adults: the global asthma insights and reality surveys. J Allergy Clin Immunol. 2004; 114(1): 40-7. http://dx.doi.org/10.1016/j.jaci.2004.04.042

18. Neffen H, Fritscher C, Schacht FC, Levy G, Chiarella P, Soriano JB, Mechali D, et al. Asthma control in Latin America: The Asthma Insights and Reality in Latin America (AIRLA) survey. Rev Panam Saúde Publica. 2005; 17(3): 191-7. http://dx.doi.org/10.1590/S1020-49892005000300007

19. Alfradique ME, Bonolo PF, Dourado I, Lima-Costa MF, Macinko J, Mendonça CS, Oliveira VB, et al. Internações por condições sensíveis à atenção primária: a construção da lista brasileira como ferramenta para medir o desempenho do sistema de saúde (Projeto ICSAP - Brasil). Cad. Saúde Pública. 2009; 25(6): 1337-1349. http://dx.doi.org/10.1590/S0102-311X2009000600016 